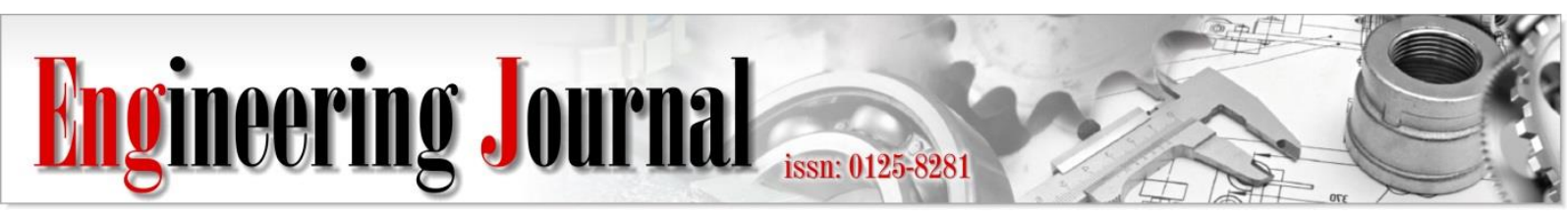

Article

\title{
Vendor Managed Inventory for Multi-Vendor Single- Manufacturer Supply Chain: A Case Study of Instant Noodle Industry
}

\author{
Huynh Tan Phong a and Pisal Yenradee ${ }^{\mathrm{b}, *}$ \\ Logistics and Supply Chain Systems Engineering Program, Sirindhorn International Institute of Technology, \\ Thammasat University, Pathum Thani 12120, Thailand \\ E-mail: ahuynhtanphong018616@gmail.com, bpisal@siit.tu.ac.th (Corresponding author)
}

\begin{abstract}
This paper develops a vendor-managed inventory (VMI) model for a multiplevendor, single-manufacturer supply chain, in which the first stage members can be traders and/or producers and the second stage member is a manufacturer. The model utilizes a realistic transportation cost which is dependent on the sizes (small- or medium-sized) of trucks. It can determine suitable sizes and numbers of trucks that minimize the transportation cost. A genetic algorithm (GA) technique, implemented in MATLAB software, is used to determine the best solution to the problem. A case study in the instant noodle industry is conducted to demonstrate the usefulness of the proposed model. Based on the experimental results, the VMI model has reasonable behaviors using sensitivity analysis. To reduce the inventory level of raw materials, the penalty cost may be set at a relatively high level or the upper inventory limits may be set at relatively low levels, without significantly affecting the average total cost per period of the entire supply chain. When the vendors are allowed to make decision independently, the solution is still the same as the solution from the proposed VMI model, which means that the manufacture does not take advantage of the vendors.
\end{abstract}

Keywords: Supply Chain Management (SCM), multi-vendor, single manufacturer, Vendor Managed Inventory (VMI), instant noodle supply chain, multiple truck sizes.

ENGINEERING JOURNAL Volume 24 Issue 6

Received 7 January 2020

Accepted 31 August 2020

Published 30 November 2020

Online at https://engj.org/

DOI:10.4186/ej.2020.24.6.91 


\section{Introduction}

With the development of global markets, supply chain management (SCM) plays an important role in incorporating different parts of a supply chain (SC) for better performances. SCM comprises planning and controlling related activities in transportation, inventory, procurement, and conversion from the upstream to the downstream members of the supply chain. SCM includes coordination and collaboration between members that can be suppliers, intermediaries, manufacturers, warehouses, third-party service providers, and final customers [1]. Walmart is an enterprise that has applied SCM successfully [2]. In SCs, inventory management has a key role in planning for production and maintaining a high service level for improving supply chain performances [1]. Solving inventory issues is a key factor that various authors want to find out polices to minimize the inventory cost and total system costs [3-7]. Vendormanaged inventory (VMI) is known as an effective inventory control strategy.

The VMI technique uses cooperating business models, in which the buyer (retailer) of a product provides specific information to a supplier (vendor) of that product. The supplier is responsible for maintaining the inventory level according to a VMI contract [8-11]. Based on a win-win relationship, the VMI contract sets frameworks, constraints about sources, work for each partner involved, and guarantees that they receive benefits. Typically, this relationship is shown in the VMI contract that vendors and retailers negotiate for the agreement of policies and benefits for each member. This improves the performances of supply chain members [10]. In previous research, the VMI model showed good performances [8] even in a seasonal demand situation [12].

This paper is motivated by three issues. First, the VMI model proposed in this paper is specially developed to be suitable for an instant noodle supply chain in Thailand, which has some characteristics that the available VMI models in the literature cannot easily model. The characteristics of this instant noodle supply chain are as follows. The first stage is multiple suppliers, which may be traders or producers while the second stage is a manufacturer of instant noodles. Moreover, transportation from the first stage to the second stage can be performed using small- and/or medium-sized trucks. The medium-sized truck has a lower unit cost for transportation than the small-sized truck. These characteristics cannot be handled by the existing VMI models. Second, the manufacturer of the instant noodles, which is the second stage of the supply chain, sets a maximum limit for each raw material to be stored at the manufacturer. When the inventory level of the raw material exceeds the preset maximum limit, the supplier of that raw material must pay a penalty cost to the manufacturer. This policy is set to control the inventory holding cost at the manufacturer. However, the maximum limit and the penalty cost are not easy to be reasonably set. The supply chain is concerned that when the maximum limit and the penalty cost are not suitably set, this may adversely affect the total cost of the entire supply chain. Therefore, the VMI model is specially developed to be suitable for the characteristics of the supply chain. In addition, the maximum limit and the penalty cost are varied. The effects on the average total cost per period of the entire supply chain are then determined. The result of this sensitivity analysis is useful for setting suitable values of the maximum limit of inventory at the manufacturer and the related penalty cost. Third, the instant noodle supply chain is dominated by the second stage which is the instant noodle manufacturer. The proposed VMI model is developed to minimize the total cost per period of the entire supply chain. It is suspected by the vendors whether they will have higher cost to follow the solution from the model when compared with making decision independently by minimizing the vendor's total cost.

This paper has specific objectives as follows.

1. To develop a VMI model that can be applied with the characteristics of the instant noodle supply chain in Thailand, which is a case study.

2. To verify that the VMI model has reasonable behaviors, using sensitivity analysis.

3. To analyze how the penalty cost and maximum limit of inventory affect the average total cost per period.

4. To analyze whether the vendors should follow the recommendation form the model or should make decision independently.

The structure of this paper is organized as follows. Section 2 reviews previous research works and explains how this paper is different from other works. Section 3 presents the concepts of the VMI model, notations of parameters and variables, and the mathematical formulas. Section 4 presents details of the GA method, which is used to solve for the best solutions to the problem. The case study data are presented in Section 5 . The results are presented and discussed in Section 6. Finally, the conclusions and recommendations for further studies are presented in Section 7.

\section{Literature Review}

The VMI technique is renowned as a tactic for inventory management, cooperation between the vendor and retailer, and balancing profits of supply chain members by sharing information [13]. Key decisions of the VMI technique are to determine when and how much to replenish the inventory. The main objective is to reduce relevant costs including transportation and inventory costs [14]. Table 1 presents the characteristics of the VMI supply chain in this paper and previous research works. From Table 1, the VMI supply chain partnership can be categorized into the single-vendor single-retailer model [10, 11, 15-17]. Some authors consider the single-vendor multi-retailer model [18-25]. 
Fewer studies consider a multi-vendor multi-retailer model $[14,26]$. This paper is different from most of the previous works in that it considers a multi-vendor, single-manufacturer supply chain, which is the structure of the instant noodle supply chain of interest.

Some previous research works consider the VMI model for the single-vendor single-retailer supply chain, in which the upstream member is a trader $[8,15,27,28]$ or the upstream member is a manufacturer $[10,11,29]$. Sadeghi, et al. [14] studied the multiple-vendor multipleretailer supply chain where the upstream members are all traders. Most research works consider that the downstream member is a trader or traders, not a manufacturer. They include $[10,11,15,30]$ for a single downstream member that is a trader. For multiple downstream members that are traders, the research works include [12, 18-20, 31]. This paper extends the VMI technique to the multiple-vendor singlemanufacturer supply chain where the upstream members may be traders and/or manufacturers, and the downstream member is a manufacturer.

There are some important issues of VMI models, including the replenishment cycle time, single inventory item or multiple inventory items, maximum inventory limit and penalty cost, and how to calculate a realistic transportation cost. The first issue to be discussed is the replenishment cycle time. Most research works assume that the replenishment cycle times of all members in the same echelon must be the same $[18,21,25]$. A few research works allow the replenishment cycle times of all members to be different $[19,20,27]$. This paper allows the upstream members to have different replenishment cycle times, making the model to be more flexible and resulting in lower average total costs per period.

Most previous research works consider only one product or inventory item to be controlled by the VMI model $[10,20,31]$. They are suitable for the supply chain of a trading business, not a manufacturing system. This paper develops the VMI model for a manufacturing supply chain, where the downstream member is an instant noodle factory that receives various raw materials from a number of vendors (both traders and manufacturers). Therefore, the supply chain involves many inventory items, which is more complicated than a single inventory item.

In the VMI model, the vendors manage when and how much to supply to the retailers. The vendors may send a large amount of product to the retailers to reduce the vendor holding cost. There is a technique to set the upper inventory limit at the retailer. If a vendor supplies beyond the upper limit, the vendor needs to pay a penalty cost (called an overstock penalty in Table 1) to the retailer. This technique restricts the quantity that the vendor delivers to the retailer, and balances the profit of the vendor and retailer in the supply chain $[18,19,21]$. This technique is different from setting the maximum storage limit at the retailers (see [14], [22]) since this is more rigid in that it does not allow the inventory level to be higher than the maximum storage limit. This paper analyzes how to set a suitable level of the upper inventory limit and the overstock penalty cost, which is rarely found in previous research works.

In the supply chain, the transportation cost is an expensive element that needs to be minimized. Most previous research works simply estimate the transportation cost per trip as a constant, which is not dependent on the transportation quantity. This paper considers a new method of computing the transportation cost that is more realistic. The transportation from the upstream to the downstream members is performed using small- and/or medium-sized trucks. The mediumsized truck has a lower unit cost for transportation than the small-sized truck. For a given transportation quantity, the vendor can consider which size of truck and how many trucks should be used to minimize the transportation cost.

\section{Mathematical Model}

The model is developed based on the following concepts.

1. The time bucket is daily.

2. The first stage of the supply chain is multiple vendors. Some vendors are producers and others are traders. The second stage of the supply chain is a manufacturer. Each vendor supplies different raw materials to the manufacturer.

3. There are two main decision variables, namely, the production cycle time of the manufacturer $(T)$ and the replenishment frequency of vendor $i\left(n_{i}\right)$. $T$ is the number of days (integer) that the manufacturer continuously produces a batch of the product. During $T$ days, vendor $i$ (the producer) continuously produces a batch of raw material with a production quantity of $Q_{i}$. This vendor adjusts the production rate to be the same as the consumption rate of the raw material at the manufacturer, to minimize the inventory level according to the lean concept. Similarly, vendor $i$ (the trader) orders a batch of raw material with an order quantity of $Q_{i}$ from its supplier during $T$ days.

4. During $T$ days, vendor $i$ delivers the raw material to the manufacturer for $n_{i}$ times, where $n_{i}$ is an integer. Therefore, the replenishment cycle time of vendor $i\left(t_{i}\right)$ is $\frac{T}{n_{i}}$. Note that $t_{i}$ may be an integer or a real number. The transportation quantity from vendor $i$ to the manufacturer per each delivery $\left(q_{i}\right)$ is $D_{i} t_{i}$, where $D_{i}$ is the demand rate of the raw material supplied by vendor $i$.

5. When the maximum inventory level of material from vendor $i$ at the manufacturer is higher than the upper limit $\left(U_{i}\right)$, vendor $i$ must pay a penalty cost [7]. 
Table 1. Comparison of previous research works and this research.

\begin{tabular}{|c|c|c|c|c|c|c|c|c|c|}
\hline \multirow[t]{2}{*}{ Paper } & \multicolumn{4}{|c|}{$\begin{array}{l}\text { Type of } \\
\text { model }^{*}\end{array}$} & \multirow{2}{*}{$1^{\text {st }}$ stage } & \multirow{2}{*}{$2^{\text {nd }}$ stage } & \multirow{2}{*}{ Decision variables } & \multirow{2}{*}{ constraints } & \multirow{2}{*}{$\begin{array}{l}\text { Solution } \\
\text { Algorithm }\end{array}$} \\
\hline & $\mathbf{A}$ & B & C & D & & & & & \\
\hline [10] & $\mathrm{X}$ & & & & $\begin{array}{l}\text { A } \\
\text { manufacturer }\end{array}$ & A trader & $\begin{array}{l}\text { Manufacturer's } \\
\text { production quantity, } \\
\text { Purchase price offered } \\
\text { to manufacturer }\end{array}$ & $\begin{array}{l}\text { Demand, Return } \\
\text { leftover product } \\
\text { to manufacturer }\end{array}$ & $\begin{array}{l}\text { Analytical } \\
\text { method based } \\
\text { on expected } \\
\text { profit }\end{array}$ \\
\hline [15] & $\mathrm{X}$ & & & & A trader & A trader & $\begin{array}{l}\text { Replenishment } \\
\text { quantity }\end{array}$ & none & $\begin{array}{l}\text { Analytical } \\
\text { method based } \\
\text { on total cost }\end{array}$ \\
\hline$[20]$ & & $\mathrm{X}$ & & & $\begin{array}{l}\text { A } \\
\text { manufacturer }\end{array}$ & Traders & $\begin{array}{l}\text { Replenishment cycle } \\
\text { time }\end{array}$ & none & $\begin{array}{l}\text { Analytical } \\
\text { method based } \\
\text { on total cost }\end{array}$ \\
\hline [19] & & $\mathrm{X}$ & & & A trader & Traders & $\begin{array}{l}\text { Order quantity, } \\
\text { Delivery quantity, } \\
\text { Replenishment cycle } \\
\text { time }\end{array}$ & $\begin{array}{l}\text { Overstock } \\
\text { penalty }\end{array}$ & $\begin{array}{l}\text { Iterative } \\
\text { heuristic }\end{array}$ \\
\hline [18] & & $\mathrm{X}$ & & & A trader & Traders & $\begin{array}{l}\text { Order quantity, } \\
\text { Delivery quantity }\end{array}$ & $\begin{array}{l}\text { Overstock } \\
\text { penalty }\end{array}$ & $\begin{array}{l}\text { An algorithm } \\
\text { based on } \\
\text { KKT points }\end{array}$ \\
\hline [21] & & $\mathrm{X}$ & & & A trader & Traders & $\begin{array}{l}\text { Replenishment cycle } \\
\text { time, Delivery quantity }\end{array}$ & $\begin{array}{l}\text { Overstock } \\
\text { penalty }\end{array}$ & $\begin{array}{l}\text { Mathematical } \\
\text { programming } \\
\text { based on } \\
\text { expected total } \\
\text { cost }\end{array}$ \\
\hline [22] & & $\mathrm{X}$ & & & A trader & Traders & $\begin{array}{l}\text { Replenishment cycle } \\
\text { time, Order quantity }\end{array}$ & $\begin{array}{l}\text { Maximum } \\
\text { storage limit }\end{array}$ & GA \\
\hline [25] & & $\mathrm{X}$ & & & $\begin{array}{l}\text { A } \\
\text { manufacturer }\end{array}$ & Traders & $\begin{array}{l}\text { Replenishment cycle } \\
\text { time, Delivery } \\
\text { quantity, Purchase } \\
\text { price, Promotion level }\end{array}$ & $\begin{array}{l}\text { Overstock } \\
\text { penalty }\end{array}$ & PSO \\
\hline [12] & & & & $\mathrm{X}$ & Traders & A trader & Inventory quantity & none & $\begin{array}{l}\text { Analytical } \\
\text { method based } \\
\text { on expected } \\
\text { profit }\end{array}$ \\
\hline [14] & & & & $\mathrm{X}$ & Traders & Traders & $\begin{array}{l}\text { Replenishment cycle } \\
\text { time, Order quantity }\end{array}$ & $\begin{array}{l}\text { Maximum } \\
\text { storage limit, } \\
\text { Maximum } \\
\text { number of } \\
\text { orders per period }\end{array}$ & GA, PSO \\
\hline $\begin{array}{l}\text { This } \\
\text { paper }\end{array}$ & & & $\mathrm{X}$ & & $\begin{array}{l}\text { Traders or } \\
\text { manufacturers }\end{array}$ & $\begin{array}{l}\text { A } \\
\text { manufacturer }\end{array}$ & $\begin{array}{l}\text { Replenishment cycle } \\
\text { time, Delivery quantity, } \\
\text { Production cycle time }\end{array}$ & $\begin{array}{l}\text { Overstock penalty, } \\
\text { Different sizes of } \\
\text { trucks }\end{array}$ & GA \\
\hline
\end{tabular}

\section{A: Single to single, B: Single to multiple, C: Multiple to single, D: Multiple to multiple*}

6. There are two truck sizes for transporting raw materials from the vendors to the manufacturer: small and medium. The capacity of a medium truck is more than three times (but less than four times) the capacity of a small truck. Two trips of the small truck are cheaper than a trip of a medium truck but three trips of a small truck $i$ are more expensive than a trip of a medium truck. To transport $\left(q_{i}\right) \mathrm{kg}$ of the raw material from vendor $i$ to the manufacturer, a suitable number and size of trucks must be calculated to minimize the transportation cost.

7. The total costs under consideration include the set-up cost of the manufacturer, inventory holding cost of raw materials of the manufacturer, inventory holding cost of vendors, set-up cost of a vendor when it is a producer, ordering cost of a vendor when it is a trader, 
penalty cost of a vendor when the inventory of raw material at the manufacturer exceeds the upper limit, and transportation cost of raw materials from vendors to the manufacturer.

8. The best values of $T$ and $n_{i}$ are determined using a Genetic Algorithm (GA) in MATLAB software, to minimize the average total costs per day $T C_{p}$.
Figure 1 shows inventory patterns of raw materials supplied by vendors to the manufacturer. The inventory patterns when the vendors are producer and trader are shown on the left and right, respectively. Various formulas for total costs and individual cost components are derived, based on the inventory patterns shown in Fig. 1. Before the formulas are developed, the index, parameters, decision variables, and intermediate variables are defined.

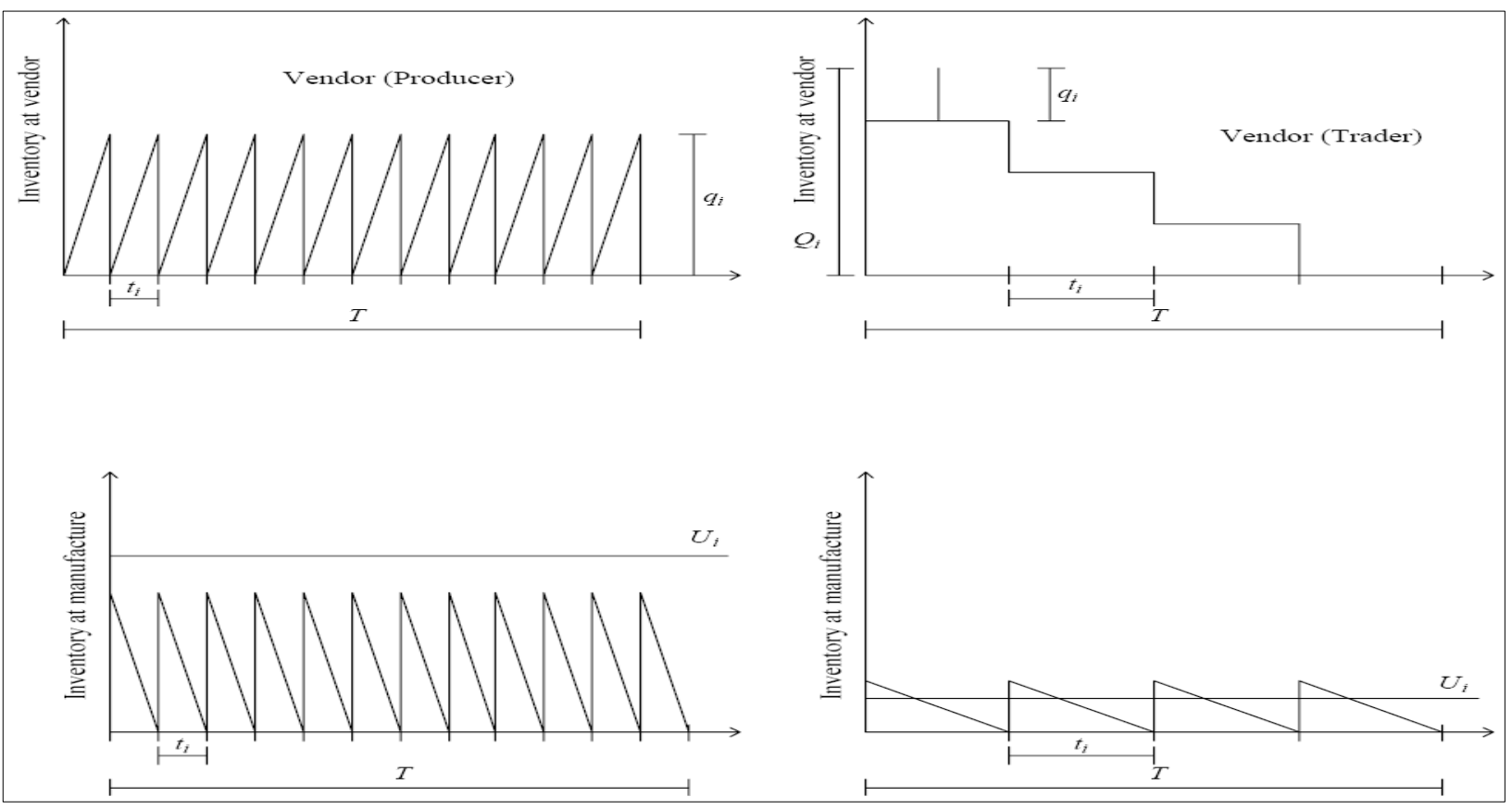

Fig. 1. Inventory pattern over time of vendors and the manufacturer.

\subsection{Index}

$i \quad$ Index of vendor, $i=(1,2,3, \ldots, m)$

\subsection{Parameters}

$m \quad$ Total number of vendors

$D_{i} \quad$ Demand rate of the material supplied by vendor $i$ (kg per day)

$C p_{m} \quad$ Capacity of medium truck $(\mathrm{kg})$

$C p_{s} \quad$ Capacity of small truck $(\mathrm{kg})$

$\mathrm{Cm}$ Transportation cost from vendor to manufacturer of medium truck (THB per trip)

Cs Transportation cost from vendor to manufacturer of small truck (THB per trip)

$a_{i} \quad$ Set-up cost of vendor $i$ when it is a producer (THB per set-up)

A Set-up cost of manufacturer (THB per set-up)

$b v_{i} \quad$ Holding cost of vendor $i$ (THB per kg-day)

$H \quad$ Holding cost of manufacturer for raw material supplied by vendor $i$ (THB per kg-day)

$\pi_{i} \quad$ Penalty cost when material supplied by vendor $i$ exceeds the upper limit at the manufacturer (THB per kg-day)

$\mathrm{O}_{i} \quad$ Ordering cost of vendor $i$ when it is a trader (THB per order)
$U_{i} \quad$ Upper limit of inventory level of material supplied by vendor $i$ at the manufacturer $(\mathrm{kg})$

$x_{i} \quad 1$ if vendor $i$ is a trader, 0 if vendor $i$ is a producer

\subsection{Decision Variables}

$n_{i} \quad$ Replenishment frequency of vendor $i$ (number of times per $T$ days)

$T \quad$ Production cycle time of manufacturer (days)

\subsection{Intermediate Variables}

Order quantity of vendor $i(\mathrm{~kg})$

$t_{i} \quad$ Replenishment cycle time of vendor $i$ (days)

$t c_{i}$ Transportation cost from vendor $i$ to manufacturer per replenishment (THB)

$q_{i} \quad$ Transportation quantity from vendor $i$ to the manufacturer $(\mathrm{kg})$

$I_{i} \quad$ Average level of inventory at vendor $i(\mathrm{~kg})$

TC Total cost per $\mathrm{T}$ periods (THB)

$T C_{p} \quad$ Total cost per period (THB per day)

TSC1 Set-up cost of the manufacturer for $T$ periods (THB)

THC1 Holding cost of the manufacturer for $T$ periods 
(THB)

TRC Transportation cost, from all vendors to the manufacturer for $T$ periods (THB)

THC2 Total holding cost of all vendors for $T$ periods (THB)

TOC Total ordering cost of all vendors for $T$ periods when they are traders (THB)

$T P C_{i} \quad$ Penalty cost of vendor $i$ for $T$ periods (THB)

TPC2 Total penalty cost of all vendors for $T$ periods (THB)

TSC2 Total set-up cost of all vendors for $T$ periods when they are producers (THB)

\subsection{Cost Formula}

The total cost per $T$ periods (TC) has a number of components, as shown in Eq. (1).

$T C=T S C 1+T H C 1+T R C+T H C 2+T O C+T P C 2+T S C 2$

Intermediate variables $t_{i}, Q_{i}$, and $q_{i}$ are defined as functions of known parameters and decision variables, as shown in Eqs. (2-5).

$$
\begin{aligned}
t_{i} & =\frac{T}{n_{i}} \\
Q_{i} & =D_{i} T \\
Q_{i} & =n_{i} q_{i} \\
q_{i} & =D_{i} t_{i}
\end{aligned}
$$

When the vendor is a trader, the vendor inventory is decreased by $q_{i}$ for each replenishment time $t$. Therefore, the average inventory level at trader $i\left(I_{i}\right)$ is derived as follows.

$I_{i}=\frac{\left(Q_{i}-q_{i}\right)+\left(Q_{i}-2 q_{i}\right)+\left(Q_{i}-3 q_{i}\right)+\ldots+\left(Q_{i}-\left(n_{i}-1\right) q_{i}\right)}{n_{i}}$

Based on Eq. (4), the average inventory is represented by Eq. (6). Similar expression can be found in [24].

$$
I_{i}=\frac{q_{i}\left(n_{i}-1\right)}{2}
$$

The total holding cost of vendor $i$ for $T$ periods $\left(\mathrm{THC}_{i}\right)$ is presented by Eq. (7). Note that the first and the second terms represent the holding cost when the vendor is a producer and a trader, respectively.

$$
T H C 2=\sum_{i=1}^{n} \frac{h v_{i} q_{i} T}{2}\left(1-x_{i}\right)+\sum_{i=1}^{n} b v_{i} I_{i} T x_{i}
$$

The set-up cost of vendor $i$ for $T$ periods when the vendor is a producer is presented by Eq. (8).

$$
\operatorname{TSC1}=\sum_{i=1}^{m} a_{i}^{*}\left(1-x_{i}\right)
$$

The ordering cost of vendor $i$ for $T$ periods when the vendor is a trader is presented by Eq. (9).

$$
\text { TOC }=\sum_{i=1}^{n} O_{i} x_{i}
$$

The penalty cost of vendor $i$ for $T$ periods is presented by Eq. (10 or 11).

$$
\operatorname{TPC}_{i}=0 \text { if } q_{i} \leq U_{i} \text {, or }
$$

$$
T P C_{i}=n_{i} * \frac{\left(q_{i}-U_{i}\right)^{2}}{2 * D_{i}} * \pi_{i} \text {, if } q_{i}>U_{i}
$$

The total penalty cost of all vendors for $T$ periods is presented by Eq. (12).

$$
T P C 2=\sum_{i=1}^{m} T P C_{i}
$$

The total set-up cost of the manufacturer for $T$ periods is presented by Eq. (13).

$$
\operatorname{TSC} 1=A
$$

The total holding cost of the manufacturer for $T$ periods is presented by Eq. (14).

$$
T H C 1=\sum_{i=1}^{m} \frac{H_{i} q_{i} T}{2}
$$

The total transportation cost from all vendors to the manufacturer for $T$ periods is presented by Eqs. (15-16).

$$
\operatorname{TRC}=\sum_{i=1}^{m} n_{i} t c_{i}
$$

where

$$
t_{i}=\left\{\begin{array}{c}
\left\lfloor\frac{q_{i}}{C_{m}}\right\rfloor * C m+C m, \\
\text { when } M O D\left(q_{i}, C p_{m}\right)>2 * C p_{s} \\
\left\lfloor\frac{q_{i}}{C_{m}}\right\rfloor * C m+2 * C s, \\
\text { when } 2 * C p_{s} \geq M O D\left(q_{i}, C p_{m}\right)>C p_{s} \\
\left\lfloor\frac{q_{i}}{C_{m}}\right\rfloor * C m+C s, \\
\text { when } \operatorname{MOD}\left(q_{i}, C p_{m}\right) \leq C p_{s}
\end{array}\right.
$$

Equation (15-16) are developed based on the conditions that two trips of a small truck are cheaper than a trip of a medium truck but three trips of a small truck are more expensive than a trip of a medium truck. The capacity of a medium truck is more than three times (but less than four times) the capacity of a small truck. An example of the transportation cost calculation is as follows. The capacity of a small truck, $C p_{s}$, is $2,000 \mathrm{~kg}$ with a transportation cost, Cs, of 2,500 THB, and the capacity of a medium truck, $C_{m}$, is $8,000 \mathrm{~kg}$ with a transportation cost, $\mathrm{Cm}$, of 6,000 THB. Table 2 provides a numerical example, to calculate $T C_{i}$ when $q_{i}$ is equal to $3000,7000,9500,11000$, and $13000 \mathrm{~kg}$.

The average total cost per period $\left(T C_{p}\right)$ is presented by Eq. (17).

$$
T C_{p}=\frac{T C}{T}
$$

\section{Solution Method}

The cost formula of $T C_{p}$ is nonlinear because its components are nonlinear. For example, $I_{i}=\frac{q_{i}\left(n_{i}-1\right)}{2}$ is nonlinear since $q_{i}$ and $n_{i}$ are decision variables. Based on Eq. (16), the transportation cost, $t c_{i}$, is not a continuous function since it has a modulo $(M O D)$ function. A noncontinuous function is not differentiable. The decision 
variables ( $T$ and $n_{i}$ ) are integer not real numbers. Therefore, the mathematical model in Section 3 is integer nonlinear programming model which cannot be solved optimally by mathematical programming software. Moreover, the model has a non-differentiable function, which cannot be solved analytically. The model can be solved by numerical methods or metaheuristics. From Table 1, some research works $[9,17,20]$ apply metaheuristics (GA and PSO) to successfully solve similar problems. Because of these reasons, this paper applies a metaheuristics instead of an analytic approach or mathematical programing approach to solve the proposed vendor-managed inventory problem.

A Genetic Algorithm (GA) is a stochastic search process based on natural selection [32]. A GA is an optimization search that starts with an initial random population. Every chromosome represents an individual in the population. In each generation, the chromosome progresses through sequential iterations called generations. The individuals are evaluated by fitness values. Offsprings are new chromosomes that are created by some operators, such as crossover and mutation. Throughout some generations, the algorithm finds the best individual [33].

The GA method, implemented in MATLAB 8.5 (R2015a), is used to solve the proposed vendor-managed inventory problem. There are some parameters to be set. Population size $N_{p}$ is the number of individuals in a generation. In this model, the crossover operator $C_{f}$ is a scattered crossover (see [34]) with the probability of crossover $C_{r}$ of 0.8 , and the mutation operator $M_{f}$ is an adaptive mutation (see [35]). Note that the scatter crossover and adaptive mutation are recommended by MATLAB software when decision variables are integers. There are examples in MATLAB library that successfully apply scatter crossover and adaptive mutation to real cases. The above-mentioned parameters are summarized in Table 3.

A flowchart of the genetic algorithm (GA) is presented in Fig. 2, and the steps of the GA process are as follows.

1. Create the initial population.

2. Calculate the fitness value of all chromosomes.

3. If the termination condition is reached, stop; otherwise go to step 4.

4. Choose the top 5\% of chromosomes with the highest fitness values, called the elite children. These children are chosen to survive to the next generation.

5. Based on the crossover rates, select the chromosomes from the current population using a "stochastic uniform" selection method on a mating pool. Apply the crossover operator (called "scattered crossover") to generate the offsprings.

6. Select the chromosomes from the current population for mutation using the default mutation rate in MATLAB software. Apply the mutation operator (called "adaptive mutation") to the selected chromosomes.

7. Offsprings from crossover and mutation, and elite children form a new generation.

8. Go to step 2.

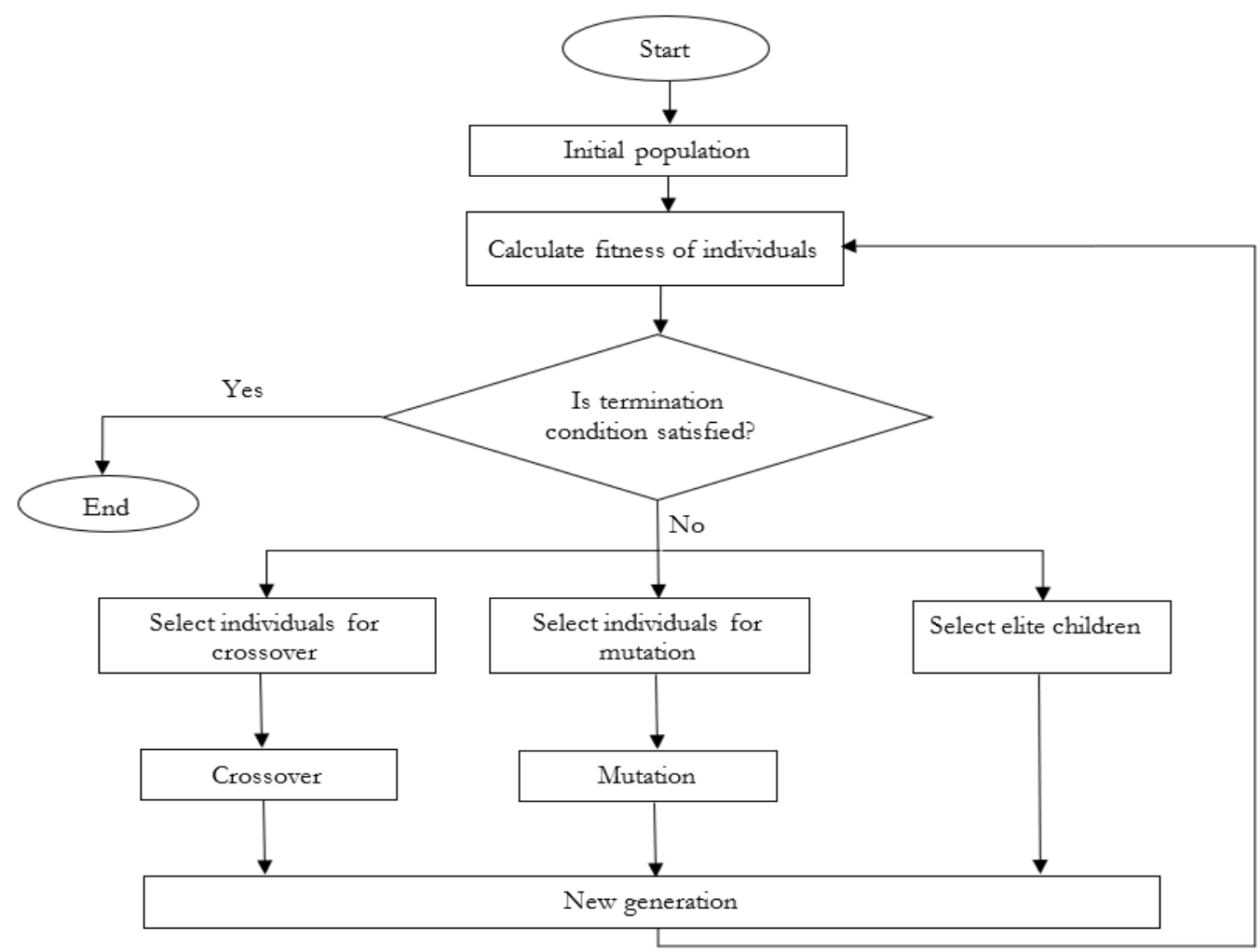

Fig. 2. Flow chart of GA method. 
Table 2. Numerical example to calculate $t c_{i}$

\begin{tabular}{|c|c|c|c|c|}
\hline$q_{i}(\mathrm{~kg})$ & $\left\lfloor\frac{q_{i}}{C p_{m}}\right.$ & $\operatorname{MOD}\left(q_{i}, C p_{m}\right)(\mathrm{kg})$ & Number of trucks & $t c_{i}(\mathrm{THB})$ \\
\hline 13,000 & 1 & 5,000 & 2 medium trucks & 12,000 \\
\hline 11,000 & 1 & 3,000 & 1 medium truck and 2 small trucks & 11,000 \\
\hline 9,500 & 1 & 1,500 & 1 medium truck and 1 small truck & 8,500 \\
\hline 7,000 & 0 & 7,000 & 1 medium truck & 6,000 \\
\hline 3,000 & 0 & 3,000 & 2 small trucks & 5,000 \\
\hline
\end{tabular}

Experiments are conducted to determine the suitable parameters (population size and number of generations) of the GA method since these parameters affect quality of solutions and computational times. Based on a guideline of MATLAB software, the population size should be set at 50 when the number of decision variables is 4 , and the number of generations should be set at 200. Three experimental cases are set with the population size, $N_{p}$, of 25,50 , and 100 . The number of generations, $G_{n}$, is fixed at 200. Each experimental case is run for 10 iterations since GA may not give the same best solution for each iteration. There are four performance indicators to be observed during the experiments, including the best solution, number of iterations that obtain the best solution (out of 10 iterations), the last generation that the solution is improved, and the computational times. Table 4 presents the performance indicators of all experimental cases.

All cases result in the same best solution $\left(T, n_{1}, n_{2}, n_{3}\right)$ $=(13,13,2,2)$. Case 1 with the population size $\left(N_{p}\right)$ of 25 obtains the best solution for only 8 iterations (out of 10 iterations). Moreover, the last generation that the solution is improved is more than 180 for some iterations. This indicates that the number of generations $\left(G_{n}\right)$ of 200 is not enough when the population size is 25 . However, the computational time of this case is the lowest. Cases 2 and 3 obtain the best solution for all iterations and the last generation that the solution is improved is less than 20. This means that Cases 2 and 3 offer reliable solution. However, Case 2 has lower computational time. Thus, the population size of 50 and number of generations of 200 is used for further analyses.

Table 3. Parameters of GA

\begin{tabular}{cc}
\hline Parameters & Value \\
\hline Population size, $N_{p}$ & 50 \\
Crossover type, $C_{f}$ & Scattered crossover \\
Crossover rate, $C_{r}$ & 0.8 \\
Mutation type, $M_{f}$ & Adaptive mutation \\
Mutation rate, $M_{r}$ & Default value of MATLAB \\
Number of generations, & software \\
$G_{n}$ & 200 \\
\hline
\end{tabular}

Table 4. Effects of GA parameters on performances

\begin{tabular}{llll}
\hline $\begin{array}{l}\text { Performance } \\
\text { indicators }\end{array}$ & $\begin{array}{l}\text { Case 1 } \\
N_{p}=25\end{array}$ & $\begin{array}{l}\text { Case 2 } \\
N_{p}=50\end{array}$ & $\begin{array}{l}\text { Case 3 } \\
N_{p}=100\end{array}$ \\
\hline $\begin{array}{l}\text { Number of } \\
\text { iterations that } \\
\text { obtain the best } \\
\text { solution }\end{array}$ & $8 / 10$ & $10 / 10$ & $10 / 10$ \\
$\begin{array}{l}\text { Last generation that } \\
\text { the solution is }\end{array}$ & $>180$ & $<20$ & $<20$ \\
$\begin{array}{l}\text { improved } \\
\text { Computational } \\
\text { time, average } \\
\text { (seconds) }\end{array}$ & 7.71 & 13.70 & 23.90 \\
$\begin{array}{l}\text { Standard deviation } \\
\text { (seconds) }\end{array}$ & 0.36 & 0.45 & 0.64 \\
\hline
\end{tabular}

\section{Case Study Data}

A real case study of an instant noodle company was conducted. However, the case study is simplified to make it easy to understand and all data are scaled to protect the confidential information of the company. The simplified case involves a supply chain with three vendors (Vendor 1 is a producer and Vendors 2 and 3 are traders) and a manufacturer. Related data are summarized in Table 5.

\section{Results and Discussion}

This section presents results and discusses managerial insights that are concluded from the results. It is divided into 4 sub-sections as follows. First, it shows the best values of decision variables, the sensitivity analysis of the $T$ variable (production cycle time of the manufacturer), how this variable affects other decision variables, the average total cost per period, and all cost components. Second, a sensitivity analysis is performed to show that the solutions from the model are reasonable. The sensitivity analysis includes the changing of vendor and manufacturer holding costs, and demand. Third, the effects of the maximum limit of the inventory level at the manufacturer, the penalty cost on the best solutions, and the average total cost per period are analyzed. It is useful to set suitable levels. Finally, the proposed VMI model that minimize the total cost of the entire supply chain is compared with the model that allow each vendor to minimize own total cost. It aims to prove whether the 
manufacturer of instant noodle take advantage of the vendors.

\subsection{The Best Solutions and Effects of T}

The GA method, implemented in MATLAB 8.5 (R2015a), is used to solve the proposed vendor-managed inventory problem. Note that there are 4 decision variables, namely, replenishment frequency of vendors $\left(n_{1}, n_{2}, n_{3}\right)$ and production cycle time of manufacturer $(T)$. When the GA is run until 200 generations, which is the termination condition, the best solution is reported. Based on 10 independent runs, the GA reports the best solution of $T=13, n_{1}=13, n_{2}=2$, and $n_{3}=2$ for all runs. This indicates that the GA parameters being used are suitable since the GA consistently generates the same solution for all runs.

There are some practical implications on the best value of $T$. When $T$ is relatively high, the manufacturer produces an instant noodle product for a large production batch before switching to produce other products. This also creates a larger inventory of products, which violates the lean concept. When $T$ is relatively low, the inventory levels of the noodle products are low. However, the manufacturer switches from one product to another more frequently. If the changeover time from one product to another is relatively long, the manufacturer may not be able to produce enough outputs to satisfy demands. Therefore, there are some situations that the best value of $T$ obtained from the model may not be used but another value is used instead. The next paragraph provides an analysis when $T$ deviates from the best value.
This part shows the sensitivity analysis of the $T$ variable, how this variable affects other decision variables, the average total cost per period, and all cost components. The $T$ value is varied from 10 to 20 , and the problem is solved to determine the best values of $n_{i}$ and all cost components. The results are shown in Table 6. It reveals that $\left(T, n_{1}, n_{2}, n_{3}\right)=(13,13,2,2)$ results in the lowest average total cost per period $\left(T C_{P}\right)$. However, if $T=13$ cannot be used because of some practical reasons, a lower value of $T$ is recommended since the average total cost per period increases less than when $T$ is higher (see Fig. 3 and Table 6). When a lower value of $T$ is selected, the supply chain operates closer to the lean concept.

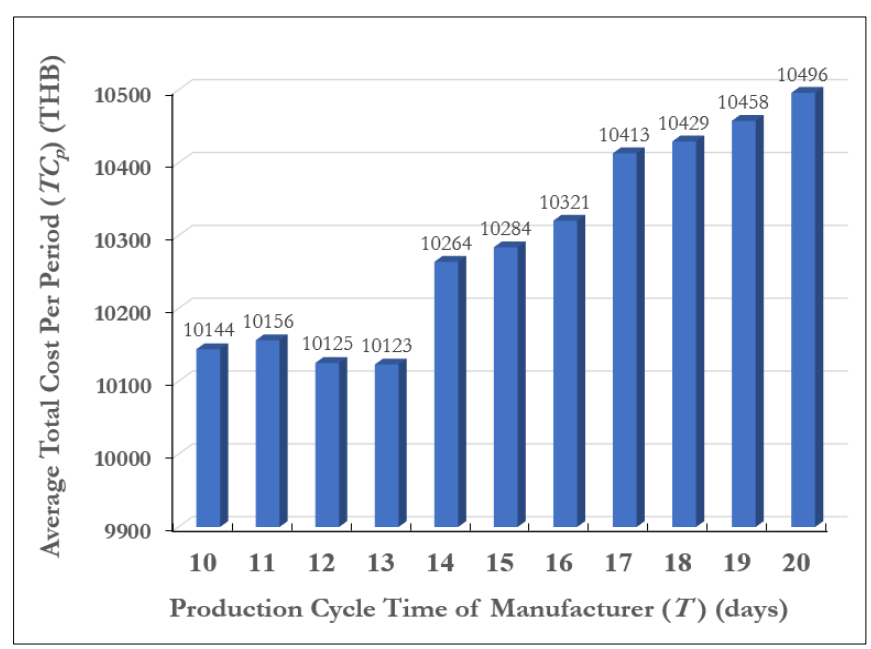

Fig. 3. Total cost per period when changing production cycle time of manufacturing $(T)$

Table 5. Data of the supply chain with three vendors and a manufacturer (base case).

\begin{tabular}{cccccccc}
\hline & $\begin{array}{c}D_{i} \\
(\mathrm{~kg} / \text { day })\end{array}$ & $\begin{array}{c}b v_{i} \\
(\mathrm{THB} / \mathrm{kg} \text {-day })\end{array}$ & $\begin{array}{c}\pi_{i} \\
(\mathrm{THB} / \mathrm{kg} \text {-day })\end{array}$ & $\begin{array}{c}a_{i} \\
(\mathrm{THB})\end{array}$ & $\begin{array}{c}H_{i} \\
(\mathrm{THB} / \mathrm{kg} \text {-day })\end{array}$ & $\begin{array}{c}O_{i} \\
(\mathrm{THB})\end{array}$ & $\begin{array}{c}U_{i} \\
(\mathrm{~kg})\end{array}$ \\
\hline Vendor 1 & 8,000 & 0.12 & 0.24 & 1250 & 0.144 & - & 24,000 \\
Vendor 2 & 1,000 & 0.12 & 0.24 & - & 0.144 & 1,000 & 3,000 \\
Vendor 3 & 300 & 0.1 & 0.2 & - & 0.12 & 1,500 & 900 \\
& $A$ & $C p_{s}$ & $C p_{m}$ & $C s$ & $C m$ & & \\
& $(\mathrm{THB})$ & $(\mathrm{kg})$ & $(\mathrm{kg})$ & $(\mathrm{THB})$ & $(\mathrm{THB})$ & & \\
& 1,500 & 2,000 & 8,000 & 2,500 & 6,000 & & \\
\hline
\end{tabular}

Table 6. Effects of $T$ on other decision variables and components of the total cost per period. *

\begin{tabular}{rrrrrrrrrrrrrrr}
\hline$T$ & $n_{1}$ & $n_{2}$ & $n_{3}$ & $q_{1}$ & $q_{2}$ & $q_{3}$ & $T H C 1 / T$ & $T H C 2 / T$ & $T O C / T$ & $T P C 2 / T$ & $T R C / T$ & $T S C 1 / T$ & $T S C 2 / T$ & $T C_{p}$ \\
\hline 10 & 10 & 5 & 2 & 8,000 & 2,000 & 1,500 & 1,035 & 810 & 250 & 24 & 7,750 & 150 & 125 & 10,144 \\
11 & 11 & 2 & 2 & 8,000 & 5,500 & 1,650 & 893 & 1,071 & 227 & 171 & 7,546 & 136 & 114 & 10,156 \\
12 & 12 & 2 & 2 & 8,000 & 6,000 & 1,800 & 930 & 1,116 & 208 & 225 & 7,417 & 125 & 104 & 10,125 \\
13 & 13 & 2 & 2 & 8,000 & 6,500 & 1,950 & 968 & 1,161 & 192 & 283 & 7,308 & 115 & 96 & 10,123 \\
14 & 14 & 2 & 3 & 8,000 & 7,000 & 1,400 & 1,040 & 1,164 & 179 & 292 & 7,393 & 107 & 89 & 10,264 \\
15 & 15 & 2 & 3 & 8,000 & 7,500 & 1,500 & 1,080 & 1,206 & 167 & 348 & 7,300 & 100 & 83 & 10,284 \\
16 & 16 & 2 & 3 & 8,000 & 8,000 & 1,600 & 1,120 & 1,248 & 156 & 406 & 7,219 & 94 & 78 & 10,321 \\
17 & 17 & 3 & 3 & 8,000 & 5,667 & 1,700 & 1,330 & 1,086 & 147 & 188 & 7,500 & 88 & 74 & 10,413 \\
18 & 18 & 3 & 3 & 8,000 & 6,000 & 1,800 & 1,380 & 1,116 & 139 & 225 & 7,417 & 83 & 69 & 10,429 \\
19 & 19 & 3 & 3 & 8,000 & 6,333 & 1,900 & 1,430 & 1,146 & 132 & 263 & 7,342 & 79 & 66 & 10,458 \\
20 & 20 & 3 & 3 & 8,000 & 6,667 & 2,000 & 1,480 & 1,176 & 125 & 303 & 7,275 & 75 & 63 & 10,496 \\
\hline
\end{tabular}

* Note that the units of $T, n_{i}, q_{i}$ are in days, unitless, and $\mathrm{kg}$, respectively. Other columns have the unit of THB. 


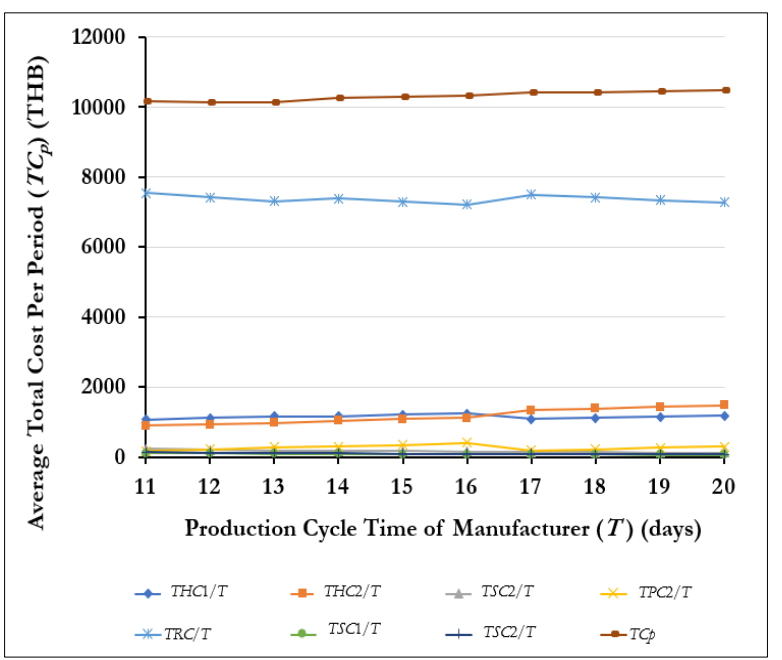

Fig. 4. Components of the total cost per period when changing $T$.

\subsection{Sensitivity Analysis to Show That the Solutions are Reasonable}

\subsubsection{Vendor holding cost}

The vendor holding cost is varied as shown in Table 7 (note that Case 2 is the base case). The results in Table 7 show that when the vendor holding costs are higher, the best total cost per day of the supply chain is increased. The entire supply chain is adjusted to have lower inventory levels by setting a shorter production cycle time of the manufacturer (lower $T$ value). The replenishment frequency of some vendors $\left(n_{i}\right)$ is also adjusted to ensure economical transportation by two truck sizes (see Fig. 5). From Fig. 5, the transportation quantity per trip from vendor 1 to the manufacturer $\left(q_{1}\right)$ is constant at 8,000 units since it is equal to the medium truck capacity. The transportation quantity from vendor $2\left(q_{2}\right)$ varies for different cases at three levels, namely 2000 , about 6,000 , and $10,000 \mathrm{~kg}$ since $2,000 \mathrm{~kg}$ is the full capacity of a small truck, $6,000 \mathrm{~kg}$ is $75 \%$ of a medium truck, and $10,000 \mathrm{~kg}$ is the full capacity of small and medium trucks. The transportation quantity from vendor $3\left(q_{3}\right)$ for Cases 4 and 5 is about 3,000 kg, which is suitable for two small trucks. This indicates that the transportation quantities are suitable for the two truck sizes and the quantities are economical.

Table 7. Effects of vendor holding cost on the best solutions and average total cost per period.*

\begin{tabular}{ccccccccc}
\hline Case & $h v_{1}$ & $h v_{2}$ & $h v_{3}$ & $T$ & $n_{1}$ & $n_{2}$ & $n_{3}$ & $T C p$ \\
\hline 1 & 0.06 & 0.06 & 0.05 & 12 & 12 & 6 & 2 & 9,562 \\
2 & 0.12 & 0.12 & 0.1 & 13 & 13 & 2 & 2 & 10,123 \\
3 & 0.18 & 0.18 & 0.15 & 12 & 12 & 2 & 2 & 10,590 \\
4 & 0.24 & 0.24 & 0.2 & 10 & 10 & 1 & 2 & 11,046 \\
5 & 0.3 & 0.3 & 0.25 & 10 & 10 & 1 & 2 & 11,260
\end{tabular}

* Note that the units of $h v_{i}, T, n_{i}$, and $T C_{p}$ are THB/kg-day, days, unitless, and THB, respectively.

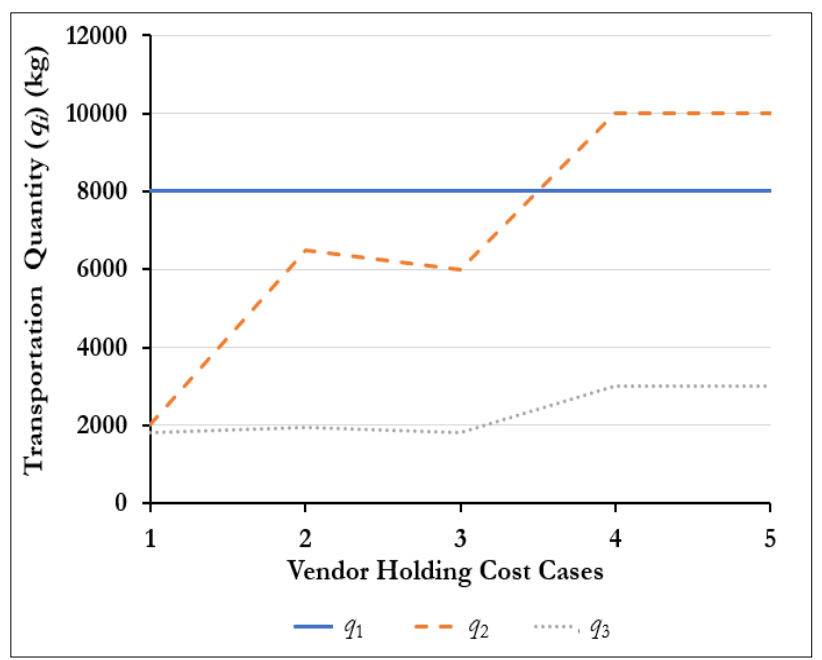

Fig. 5. Effects of vendor holding cost on transportation quantity.

Figure 6 shows how the components of the total cost per period are affected by the unit holding cost at the vendors. It reveals that the holding cost at the vendors, holding cost at the manufacturer, transportation cost, and penalty cost are affected. The reasons for this change are explained as follows. When the transportation quantities from some vendors (to the manufacturer) are changed, this directly affects the transportation cost and indirectly affects the penalty cost. A higher transportation quantity from the vendor to the manufacturer tends to create more inventory of raw material at the manufacturer, and when it exceeds the maximum limit, the penalty cost is charged to the vendor.

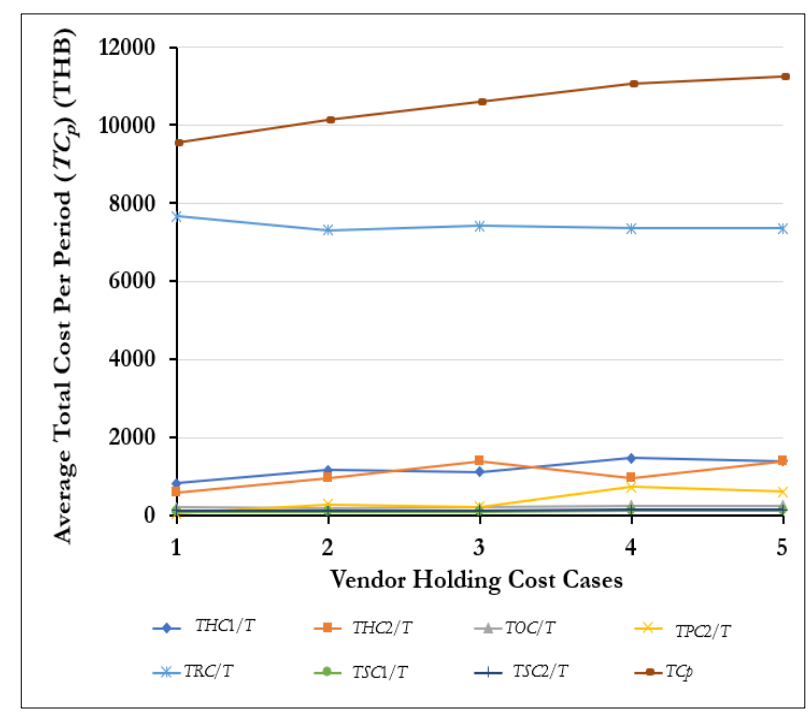

Fig. 6. Average total cost per period when changing the vendor holding cost

\subsubsection{Manufacturer holding cost}

To determine the effects of the manufacturer holding cost, the manufacturer holding cost is varied as shown in Table 8 . Note that Case 2 is the base case. Table 8 shows that the average total cost per period is 
significantly higher when the unit holding cost at the manufacturer is higher. This result is similar to the case of changing the vendor holding cost. To compensate for the higher unit holding cost at the manufacturer, the manufacturer production cycle time $(T)$ is lower to reduce the inventory level of raw materials at the manufacturer.

Table 8. Effects of manufacturer holding cost on the best solutions and average total cost per period*

\begin{tabular}{rcccccccc}
\hline Case & $H_{1}$ & $H_{2}$ & $H_{3}$ & $T$ & $n_{1}$ & $n_{2}$ & $n_{3}$ & $T C_{p}$ \\
\hline 1 & 0.072 & 0.072 & 0.06 & 13 & 13 & 2 & 2 & 9,542 \\
2 & 0.144 & 0.144 & 0.12 & 13 & 13 & 2 & 2 & 10,123 \\
3 & 0.216 & 0.216 & 0.18 & 12 & 12 & 6 & 2 & 10,561 \\
4 & 0.288 & 0.288 & 0.24 & 12 & 12 & 6 & 2 & 10,975 \\
5 & 0.36 & 0.36 & 0.3 & 10 & 10 & 5 & 2 & 11,235
\end{tabular}

* Note that the units of $H_{i}, T, n_{i}$, and $T C_{p}$ are $\mathrm{THB} / \mathrm{kg}$ day, days, unitless, and THB, respectively.

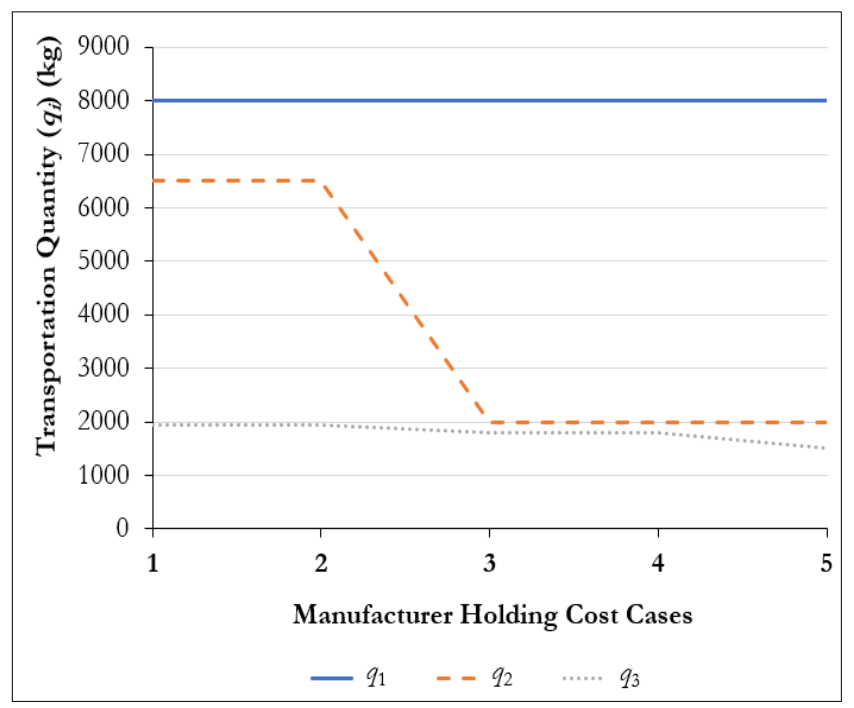

Fig. 7. Effects of manufacturer holding cost on transportation quantities.

Figure 7 shows that the transportation quantity from vendor 2 to the manufacturer is reduced from more than 6,000 kg (Cases 1 and 2) to only 2,000 kg (Cases 3, 4, and $5)$, to reduce the inventory of raw material from vendor 2 at the manufacturer. However, the transportation cost is increased since $6,000 \mathrm{~kg}$ is transported by a medium truck and 2,000 kg is transported by a small truck, which has a higher unit cost. Figure 8 shows that the average total cost per period is increased since the holding cost at the manufacturer and the transportation cost are higher.

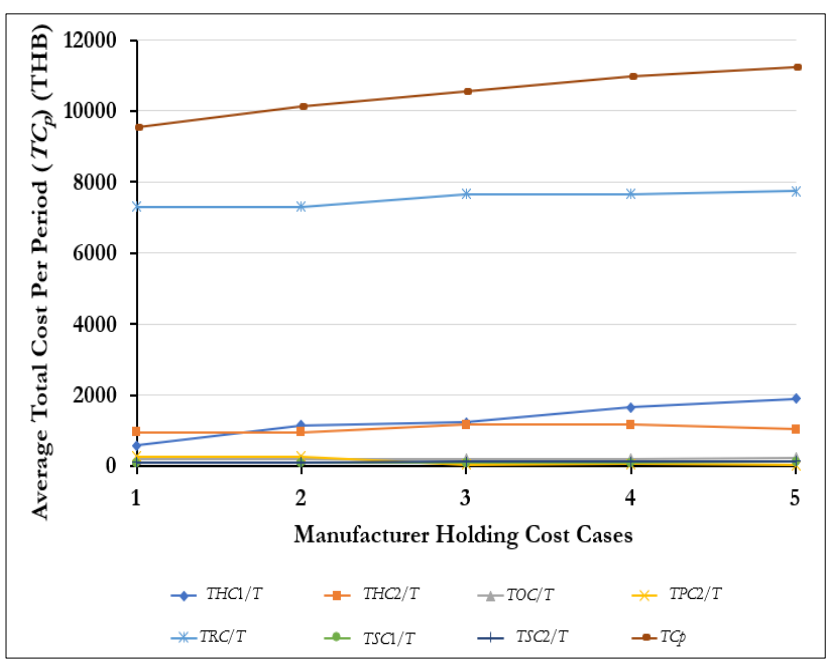

Fig. 8. Average total cost per period when changing the manufacture holding cost.

\subsubsection{Demand}

The effects of demand on the best solutions and the average total cost per period are presented in Table 9. Note that Case 3 is the base case. When the demand increases due to business growth, the proposed model suggests a reduction in the manufacturer production cycle time ( $T$ ) and an adjustment to the replenishment frequencies of vendors, to minimize the average total cost per period. Figure 9 shows the best transportation quantities from all vendors to the manufacturer, and Fig. 10 shows the components of the average total cost per period. From Fig. 10, the average total cost per period is mainly affected by the increasing transportation cost when demand increases. Figure 9 shows that when the demand increases, the transportation quantities from some vendors to the manufacturer tend to increase. For example, the transportation quantity from vendor 2 increases from 2,000 kg (using a small truck) to $6,000 \mathrm{~kg}$ (using a medium truck), and transportation quantity from vendor 3 increases from 1,000 kg to 2,000 kg (both cases use a small truck). Note that the transportation quantity from vendor 1 does not increase since $8,000 \mathrm{~kg}$ is the capacity of a medium truck. Increasing the transportation quantity slightly beyond 8,000 kg results in a significantly higher cost.

Table 9. Effects of demand on the best solution and total cost per period*

\begin{tabular}{ccccccccc}
\hline Case & $D_{1}$ & $D_{2}$ & $D_{3}$ & $T$ & $n_{1}$ & $n_{2}$ & $n_{3}$ & $T C_{p}$ \\
\hline 1 & 6,000 & 800 & 200 & 12 & 9 & 5 & 2 & 8,190 \\
2 & 7,000 & 900 & 250 & 12 & 11 & 2 & 2 & 9,392 \\
3 & 8,000 & 1000 & 300 & 13 & 13 & 2 & 2 & 10,123 \\
4 & 9,000 & 1100 & 350 & 11 & 13 & 2 & 2 & 11,368 \\
5 & 10,000 & 1,200 & 400 & 10 & 13 & 2 & 2 & 12,293 \\
\hline
\end{tabular}

* Note that the units of $D_{i}, T, n_{i}$, and $T C_{p}$ are $\mathrm{kg} / \mathrm{day}$, days, unitless, and $\mathrm{THB}$, respectively. 


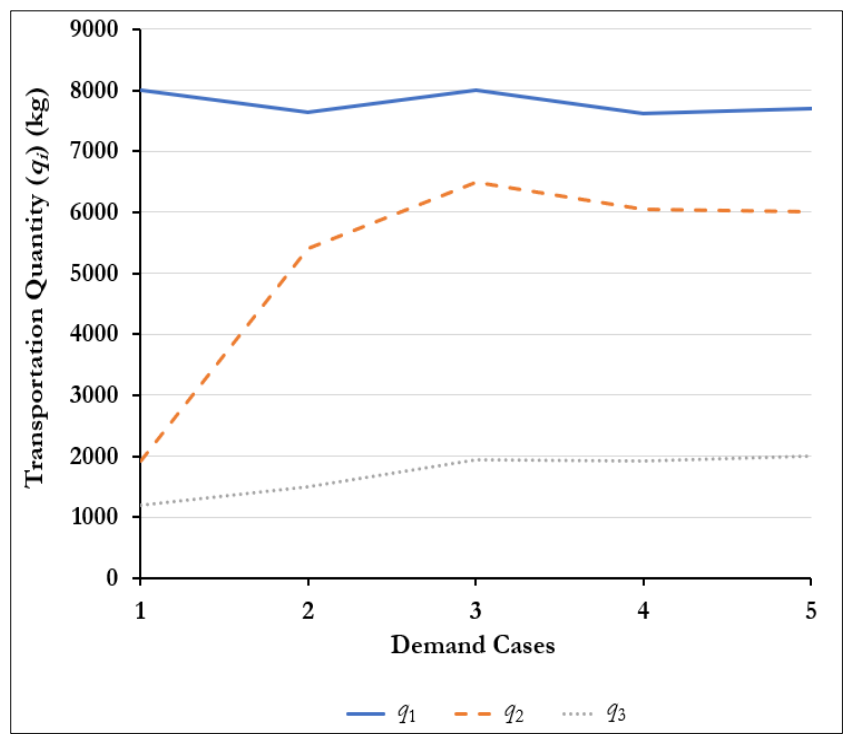

Fig. 9. Effects of demand on transportation quantity.

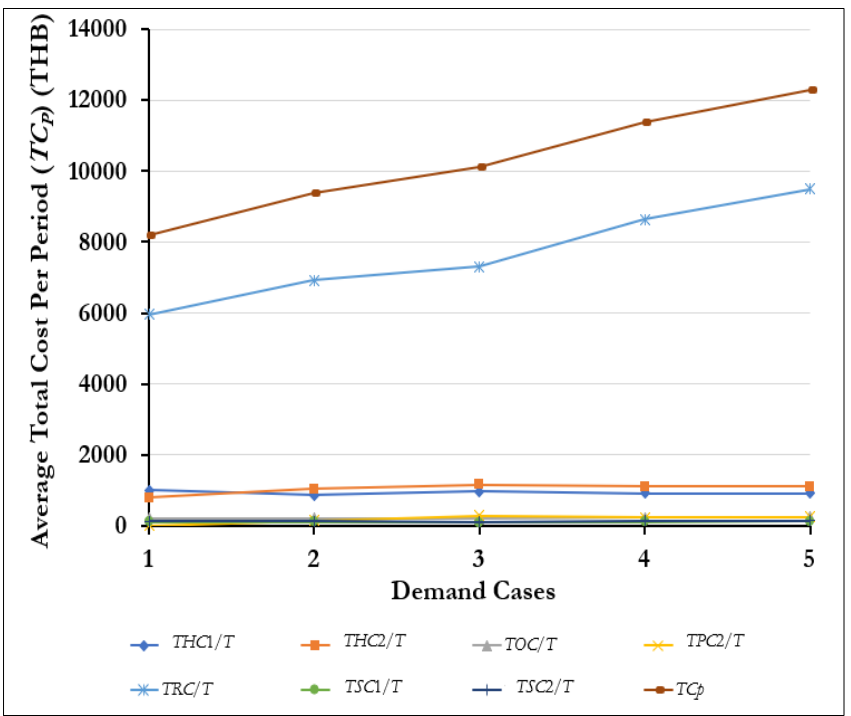

Fig. 10. Total cost per period when changing demand.

\subsection{Analysis of Upper Limit of Inventory Level at the Manufacturer and the Penalty Cost}

The section is provided to analyze the effects of the maximum limit of the inventory level at the manufacturer and the penalty cost on the best solutions and the average total cost per period. It is useful to set suitable levels.

\subsubsection{Penalty cost}

To evaluate the effects of unit penalty cost, it is varied as shown in Table 10. Case 2 is the base case. Table 10 shows that when the penalty cost is increased, the model suggests a reduction in the production cycle time at the manufacturer $(T)$ and an adjustment of the frequency of replenishment of vendors, to reduce transportation quantities from some vendors to the manufacturer as shown in Figs. 11-13. When the transportation quantities are reduced, the inventory level of raw materials at the manufacturer is also reduced. This results in a lower amount of inventory that exceeds the maximum limit. This limits the incurred total penalty cost to a reasonable level although the unit penalty cost is higher. Figure 11 shows that vendor 1 has no penalty cost. Figure 12 shows that the transportation quantity from vendor 2 is reduced until it does not exceed the maximum limit. Figure 13 shows that the transportation quantity from vendor 3 is reduced, to control the amount that exceeds the maximum limit.

Table 10 and Figure 14 show that the average total cost per period of the supply chain system is only slightly increased when the unit penalty cost is higher. The degree that the average total cost per period increases is not as high as when the holding cost at the manufacturer or vendor increases (see Figs. 6 and 8).

Table 10. Effects of penalty cost on the best solutions and average total cost per period.*

\begin{tabular}{ccccccccc}
\hline $\begin{array}{c}\text { Cas } \\
\mathrm{e}\end{array}$ & $\pi_{1}$ & $\pi_{2}$ & $\pi_{3}$ & $T$ & $n_{1}$ & $n_{2}$ & $n_{3}$ & $T C_{p}$ \\
\hline 1 & 0.12 & 0.12 & 0.1 & 13 & 13 & 2 & 2 & 9,981 \\
2 & 0.24 & 0.24 & 0.2 & 13 & 13 & 2 & 2 & 10,123 \\
3 & 0.36 & 0.36 & 0.3 & 10 & 10 & 5 & 2 & 10,156 \\
4 & 0.48 & 0.48 & 0.4 & 10 & 10 & 5 & 2 & 10,168 \\
5 & 0.6 & 0.6 & 0.5 & 10 & 10 & 5 & 2 & 10,180 \\
\hline
\end{tabular}

* Note that the units of $\pi_{i}, T, n_{i}$, and $T C_{p}$ are $\mathrm{THB} / \mathrm{kg}-$ day, days, unitless, and THB, respectively.

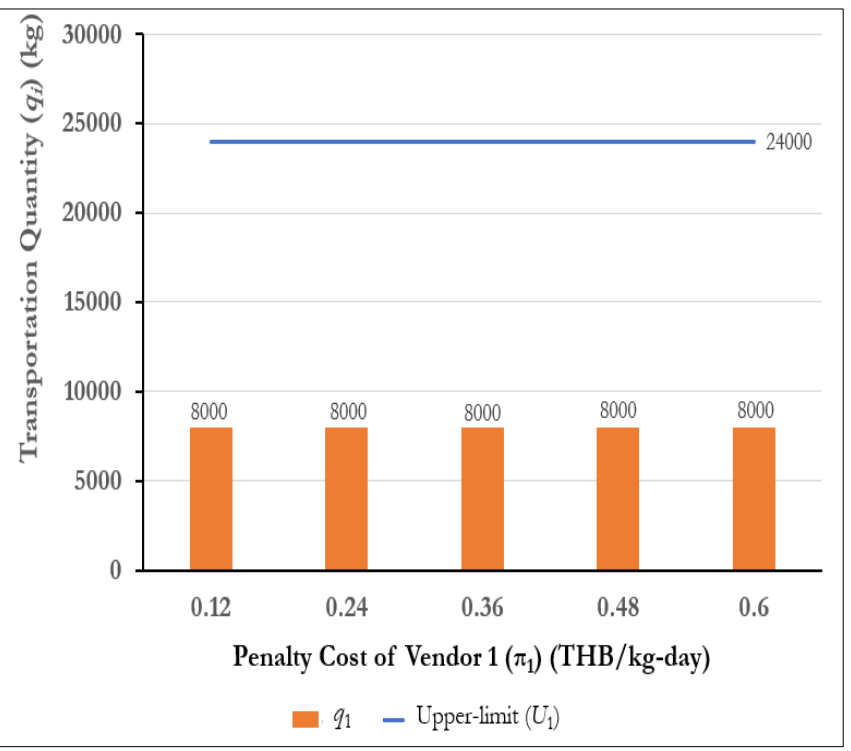

Fig. 11. Effects of penalty cost on transportation quantity of vendor 1 . 


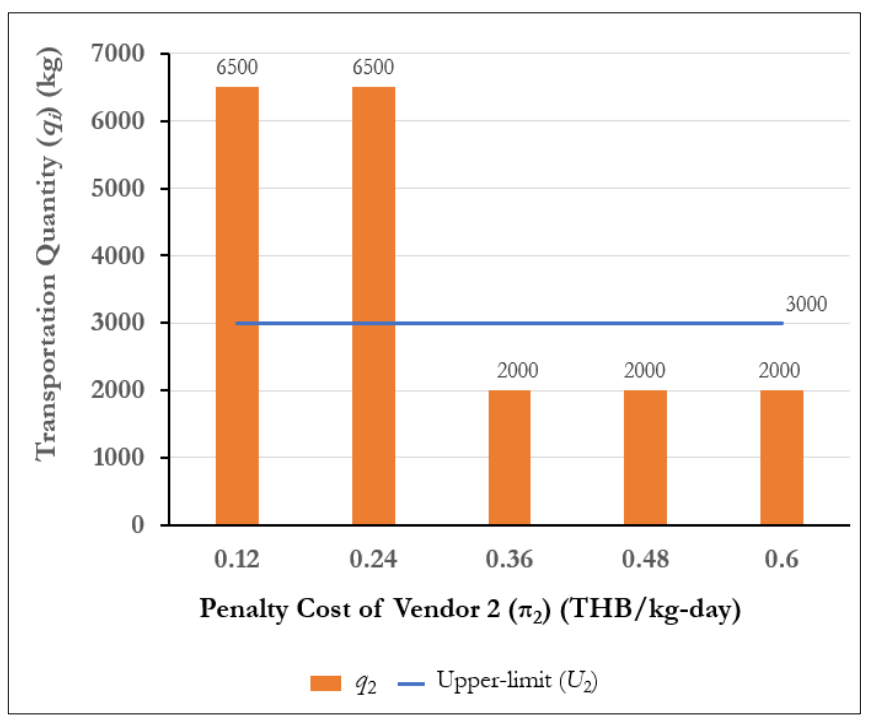

Fig. 12. Effects of penalty cost on transportation quantity of vendor 2 .

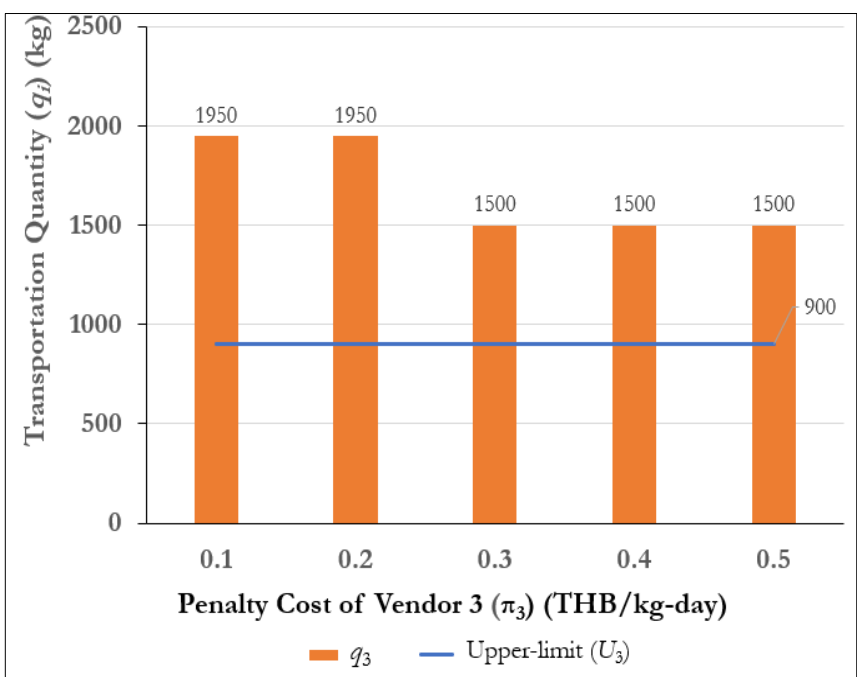

Fig. 13. Effects of penalty cost on transportation quantity of vendor 3 .

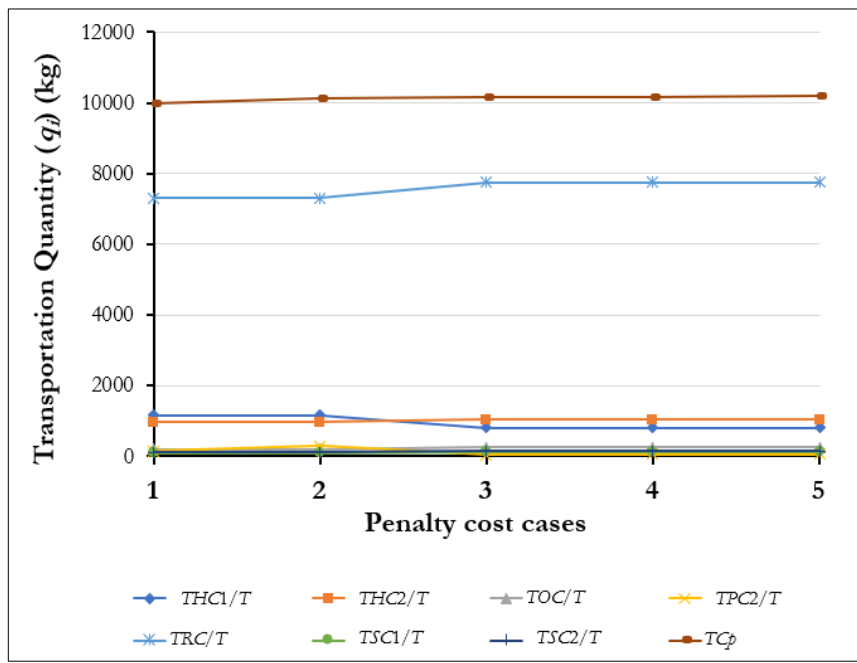

Fig. 14. Average total cost per period when changing penalty cost.

\subsubsection{Upper limit of inventory at the manufacturer}

When the manufacturer wants to reduce the inventory level of raw materials, it sets the upper limit of inventory to be lower. This experiment is conducted to see whether it is possible to manage the VMI system so that the average total cost per period is not significantly increased when the upper limit of inventory is reduced.

Table 11 shows five cases where the upper limit of the inventory level is reduced from the base case (Case 1). It reveals that the proposed model can suggest suitable values for the production cycle time of the manufacturer $(T)$ and the replenishment frequency of vendors $\left(n_{i}\right)$, to stabilize the average total cost per period $\left(T C_{p}\right)$ as shown in Fig. 16. Figure 15 shows that the transportation quantity from vendor 2 is significantly reduced from more than $6,000 \mathrm{~kg}$ to $2,000 \mathrm{~kg}$, reducing the penalty cost and also reducing the holding cost of the raw material at the manufacturer (see Fig. 16). However, the transportation cost (see Fig. 16) is higher since a small truck is used instead of a medium truck. Similarly, the transportation quantity from vendor 3 is slightly reduced (see Fig. 15).

Table 11. Effects of the upper limit of inventory at the manufacture on the average total cost per period.*

\begin{tabular}{ccccccccc}
\hline Case & $U_{1}$ & $U_{2}$ & $U_{3}$ & $T$ & $n_{1}$ & $n_{2}$ & $n_{3}$ & $T C_{p}$ \\
\hline 1 & 24,000 & 3,000 & 900 & 13 & 13 & 2 & 2 & 10,123 \\
2 & 22,000 & 2,600 & 800 & 10 & 10 & 5 & 2 & 10,153 \\
3 & 20,000 & 2,200 & 700 & 10 & 10 & 5 & 2 & 10,163 \\
4 & 18,000 & 1,800 & 600 & 10 & 10 & 5 & 2 & 10,176 \\
5 & 16,000 & 1,400 & 500 & 10 & 10 & 5 & 2 & 10,208
\end{tabular}

* Note that the units of $U_{i}, T, n_{i}$, and $T C_{p}$ are $\mathrm{kg}$, days, unitless, and THB, respectively.

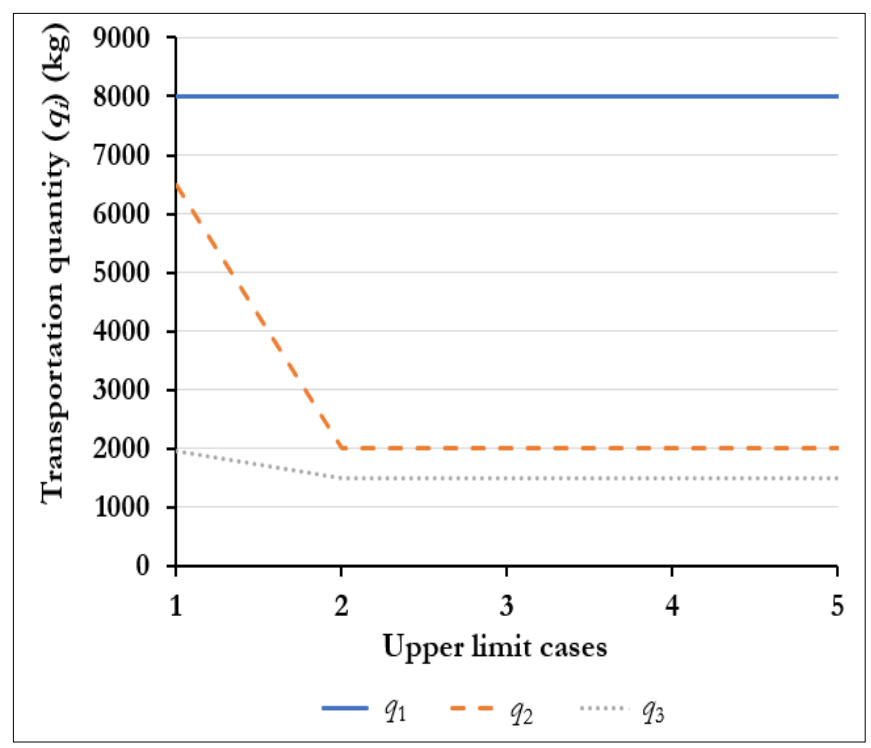

Fig. 15. Effects of the upper limit of inventory at the manufacture on transportation quantity. 


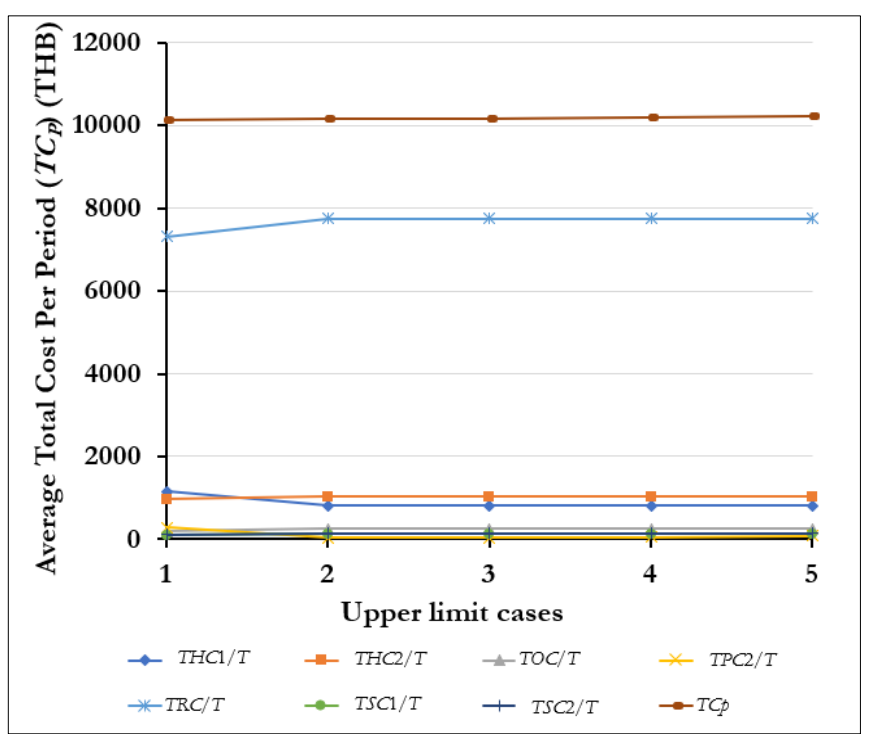

Fig. 16. Average total cost per period when changing the upper limit of inventory at the manufacturer.

\subsection{Optimizing the Entire Supply Chain vs. Individual Member}

The proposed VMI model optimizes the total cost per period of the entire supply chain and the best solution is $\left(T, n_{1}, n_{2}, n_{3}\right)=(13,13,2,2)$ with the best total cost per period of 10,123 Baht as shown in Table 6 .

A sub-model is constructed considering only total cost of each vendor and allow each vendor to solve for the best value of $n_{i}$ independently. In the sub-model, it has a parameter, which is the production cycle time of manufacturer $(T) . T$ is fixed at 13 because it is the best value suggested by the proposed VMI model and $T$ is decided by the manufacturer. When the sub-model is solved independently for each vendor, the best solution is obtained with $\left(n_{1}, n_{2}, n_{3}\right)=(13,2,2)$. This result indicates that when each vendor is allowed to make decision independently to optimize own total cost. The solution is the same as the solution obtained by the proposed VMI model that minimize total cost of the entire supply chain. It concludes that the proposed VMI model does not force vendors to scarify to maximize benefit of the entire supply chain based on the current cost structure of this case study.

\section{Conclusions and Further Studies}

This paper develops a vendor-managed inventory model to satisfy the special characteristics of the instant noodle supply chain in Thailand, which has not been addressed before by past works. The special characteristics are as follows. The first stage is multiple suppliers, which may be traders or producers while the second stage is a manufacturer of instant noodles. Moreover, transportation from the first stage to the second stage can be performed using small- and/or medium-sized trucks. A medium-sized truck has a lower unit cost of transportation than a small-sized truck. These characteristics cannot be handled by the existing VMI models.

Data of the case study is collected from an instant noodle company in Thailand. The developed VMI model is applied to the instant noodle supply chain, and the experimental results are reported. The experimental results reveal that the proposed VMI model can determine reasonable solutions and suggest how to effectively manage the VMI system.

This paper has both theoretical and practical contributions. The theoretical contributions are as follows.

1. The proposed VMI model is specially developed to match the characteristics of the instant noodle supply chain in Thailand, which cannot be handled directly by the available VMI models in the literature.

2. Although the proposed VMI model is developed for a single product, it can be easily extended to handle multiple products, given that the production capacity is sufficient. For example, product $\mathrm{A}$ is produced by the manufacturer for 13 days $(T=13)$ in a month. Then, the manufacturer switches to produce product $B$ for 10 days $(T=10)$ in that month. Therefore, the VMI problem for producing products $A$ and $B$ by this supply chain can be considered and solved separately.

The practical contributions of this paper are managerial insights obtained from the experimental results, which are presented as follows.

1. The sensitivity analysis of the $T$ variable, how this variable affects other decision variables, the average total cost per period, and all cost components presented in Section 6.1 are useful for the instant noodle supply chain. This indicates that setting the best value of the production cycle time of the manufacturer $(T)$ results in the lowest average total cost per period. However, when the $T$ value is reduced from the best value, the average total cost per period is slightly increased. In contrast, when the $T$ value is increased, the average total cost per period is highly increased. Therefore, the supply chain is motivated to implement the lean concept by reducing the $T$ value to reduce the production batch size and raw material inventory at the manufacturer, which is the main member of the instant noodle supply chain.

2. The transportation cost is the highest cost component of the supply chain in the case study, as shown in Sections 6.1 to 6.3. Most companies in Thailand use trucks with multiple sizes for 
3. transportation, dependent on the amount of materials. Trucks of different sizes have a different transportation cost per trip. The proposed VMI model allows trucks of different sizes to be used. Therefore, the transportation cost in the model is much more accurate and practical than a model with only one truck size. This issue is the major practical contribution of the proposed VMI model.

4. The effects of the penalty cost and the upper limit of raw material inventory at the manufacturer on the best solutions and cost components (presented in Section 6.3) are beneficial for the supply chain. Setting different penalty costs and upper limits do not significantly affect the average total cost per period of the supply chain since the proposed VMI model is effective for determining the best decision variables $\left(T\right.$, and $\left.n_{i}\right)$, to minimize the average total cost per period. Therefore, when the manufacturer would like to reduce the inventory level of raw materials, the penalty cost may be set at a relatively high level or the upper

\section{References}

[1] A. Harrison and R. I. V. Hoek, Logistics Management and Strategy: Competing through the Supply Chain, 5th ed. Pearson Education, 2008.

[2] L.-X. Xu, Q. Xu, and X. Liu, "Wal-Mart and Carrefour's supply chain management strategies in China," International Journal of Business and Management, vol. 9, p. 155, 2014.

[3] V. Pukcarnon, P. Chaovalitwongse, and N. Phumchusri, "The can-order policy for onewarehouse $\mathrm{N}$-retailer inventory system: a heuristic approach," Engineering Journal, vol. 18, pp. 53-72, 2014.

[4] S. Kositphon and P. Chaovalitwongse, "An application of systems thinking for solving inventory management problems," Engineering Journal, vol. 20, pp. 67-88, 2016.

[5] Q. Wang, N. Zhao, J. Wu, and Q. Zhu, "Optimal pricing and inventory policies with reference price effect and loss-averse customers," Omega, p. 102174, 2019.

[6] N. Sakulsom and W. Tharmmaphornphilas, "Periodic-review policy for a 2-echelon inventory problem with seasonal demand," Engineering Journal, vol. 22, pp. 117-134, 2018.

[7] L. Janssen, A. Diabat, J. Sauer, and F. Herrmann, "A stochastic micro-periodic age-based inventory replenishment policy for perishable goods," Transportation Research Part E: Logistics and Transportation Review, vol. 118, pp. 445-465, 2018. limits may be set at relatively low levels, without significantly affecting the average total cost per period of the entire supply chain.

5. The proposed VMI model that minimizes total cost of the entire supply chain give the solution which is the same as the solution form the model that minimize cost of vendors independently. Thus, the manufacturer of the instant noodle supply chain does not take advantage of the vendors.

The proposed VMI model is limited in that it needs crisp or constant parameters. However, the customer demand for the instant noodle company is affected by its marketing promotions and competitor promotions. Thus, this is a highly uncertain environment. A further study is needed to extend the VMI model to handle uncertain or fuzzy demand situations. The fuzzy demand under pessimistic, most likely, and optimistic situations should be estimated. An extended VMI model should be developed to determine a robust solution that maximizes the expected total cost per period of all situations.

[8] R. Guan and X. Zhao, "On contracts for VMI program with continuous review $(\mathrm{r}, \mathrm{Q})$ policy," European Journal of Operational Research, vol. 207, pp. 656-667, 2010.

[9] J. Shu, Z. Li, H. Shen, T. Wu, and W. Zhong, "A logistics network design model with vendor managed inventory," International Journal of Production Economics, vol. 135, pp. 754-761, 2012.

[10] C. H. Huynh and W. Pan, "Operational strategies for supplier and retailer with risk preference under VMI contract," International Journal of Production Economics, vol. 169, pp. 413-421, 2015.

[11] H. Yu, L. Tang, Y. Xu, and Y. Wang, "How much does VMI better than RMI in a global environment?," International Journal of Production Economics, vol. 170, pp. 268-274, 2015.

[12] J. Cai, P. R. Tadikamalla, J. Shang, and G. Huang, "Optimal inventory decisions under vendor managed inventory: Substitution effects and replenishment tactics," Applied Mathematical Modelling, vol. 43, pp. 611-629, 2017.

[13] Y. Dong, M. Dresner, and Y. Yao, "Beyond information sharing: An empirical analysis of vendor-managed inventory," Production and Operations Management, vol. 23, pp. 817-828, 2014.

[14] J. Sadeghi, S. M. Mousavi, S. T. A. Niaki, and S. Sadeghi, "Optimizing a multi-vendor multi-retailer vendor managed inventory problem: Two tuned meta-heuristic algorithms," Knowledge-Based Systems, vol. 50, pp. 159-170, 2013.

[15] Y. Yao, P. T. Evers, and M. E. Dresner, "Supply chain integration in vendor-managed inventory," Decision Support Systems, vol. 43, pp. 663-674, 2007. 
[16] B. Hu, C. Meng, D. Xu, and Y.-J. Son, "Supply chain coordination under vendor managed inventory-consignment stocking contracts with wholesale price constraint and fairness," International Journal of Production Economics, vol. 202, pp. 21-31, 2018.

[17] A. Sainathan and H. Groenevelt, "Vendor managed inventory contracts-coordinating the supply chain while looking from the vendor's perspective," European Journal of Operational Research, vol. 272, pp. 249-260, 2019.

[18] M. A. Darwish and O. M. Odah, "Vendor managed inventory model for single-vendor multi-retailer supply chains," European Journal of Operational Research, vol. 204, pp. 473-484, 2010.

[19] M. Hariga, M. Gumus, A. Daghfous, and S. K. Goyal, "A vendor managed inventory model under contractual storage agreement," Computers \& Operations Research, vol. 40, pp. 2138-2144, 2013.

[20] A. Mateen and A. K. Chatterjee, "Vendor managed inventory for single-vendor multi-retailer supply chains," Decision Support Systems, vol. 70, pp. 31-41, 2015.

[21] A. Mateen, A. K. Chatterjee, and S. Mitra, "VMI for single-vendor multi-retailer supply chains under stochastic demand," Computers \& Industrial Engineering, vol. 79, pp. 95-102, 2015.

[22] J. Sadeghi and S. T. A. Niaki, "Two parameter tuned multi-objective evolutionary algorithms for a biobjective vendor managed inventory model with trapezoidal fuzzy demand," Applied Soft Computing, vol. 30, pp. 567-576, 2015.

[23] Z. Belalia and F. Ghaiti, "The value of vendor managed inventory in an autocorrelated demand environment," IFAC-PapersOnLine, vol. 49, pp. 668673, 2016.

[24] M. A. Kaasgari, D. M. Imani, and M. Mahmoodjanloo, "Optimizing a vendor managed inventory (VMI) supply chain for perishable products by considering discount: Two calibrated meta-heuristic algorithms," Computers \& Industrial Engineering, vol. 103, pp. 227-241, 2017.

[25] Z. Chen, "Optimization of production inventory with pricing and promotion effort for a singlevendor multi-buyer system of perishable products," International Journal of Production Economics, vol. 203, pp. 333-349, 2018.
[26] T. Poorbagheri, "Vendor managed inventory of a multiple-vendor multiple-retailer supply chain with stochastic demand and unequal replenishment cycle times," in IIEC International Industrial Engineering Conference, 2015.

[27] M. Hariga, M. Gumus, and A. Daghfous, "Storage constrained vendor managed inventory models with unequal shipment frequencies," Omega, vol. 48, pp. 94-106, 2014.

[28] J.-Y. Lee and R. K. Cho, "Optimal (z, Z)-type contracts for vendor-managed inventory," International Journal of Production Economics, vol. 202, pp. 32-44, 2018.

[29] M. Khan, M. Y. Jaber, S. Zanoni, and L. Zavanella, "Vendor managed inventory with consignment stock agreement for a supply chain with defective items," Applied Mathematical Modelling, vol. 40, pp. 7102-7114, 2016.

[30] J.-Y. Lee, R. K. Cho, and S.-K. Paik, "Supply chain coordination in vendor-managed inventory systems with stockout-cost sharing under limited storage capacity," European Journal of Operational Research, vol. 248, pp. 95-106, 2016.

[31] K. Govindan, "The optimal replenishment policy for time-varying stochastic demand under vendor managed inventory," European Journal of Operational Research, vol. 242, pp. 402-423, 2015.

[32] S. H. R. Pasandideh, S. T. A. Niaki, and A. R. Nia, "A genetic algorithm for vendor managed inventory control system of multi-product multi-constraint economic order quantity model," Expert Systems with Applications, vol. 38, pp. 2708-2716, 2011.

[33] S. H. R. Pasandideh and S. T. A. Niaki, "A genetic algorithm approach to optimize a multi-products EPQ model with discrete delivery orders and constrained space," Applied Mathematics and Computation, vol. 195, pp. 506-514, 2008.

[34] D. Hakimi, D. Oyewola, Y. Yahaya, and G. Bolarin, "Comparative analysis of genetic crossover operators in knapsack problem," Journal of Applied Sciences and Environmental Management, vol. 20, pp. 593-596, 2016.

[35] A. B. Djurišić, "Elite genetic algorithms with adaptive mutations for solving continuous optimization problems - application to modeling of the optical constants of solids," Optics Communications, vol. 151, pp. 147-159, 1998. 


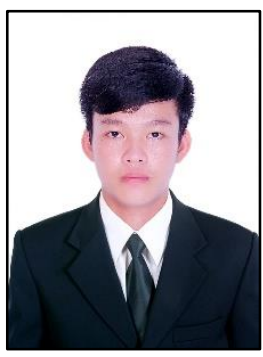

Huynh Tan Phong was born in O Mon, Can Tho, Vietnam in 1995. He received a B.S. degree in Industrial Management from Can Tho University, in 2013. He has been studying for an M.S. degree in Logistics and Supply Chain Systems Engineering from Sirindhorn International Institute of Technology, Thammasat University, Pathum-Thani 12121, Thailand since 2017.

From 2013 to 2017, he was a B.S student in Can Tho University with graduation research related to risk management in a seafood supply chain. In 2017, He got an Excellent Foreign Student (EFS) scholarship to study for an M.S in Sirindhorn International Institute of Technology (SIIT), Thammasat University.

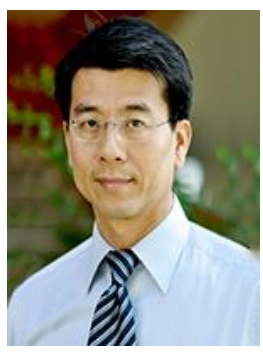

Pisal Yenradee is an Associate Professor in the industrial engineering program at the Sirindhorn International Institute of Technology (SIIT), Thammasat University. He holds a B.Eng. in production engineering from King Mongkut's Institute of Technology and an M.Eng. and D.Eng. in industrial engineering and management from the Asian Institute of Technology. His research interests focus on production planning and inventory control systems, JIT, ERP, TOC, finite capacity scheduling systems, aggregate production planning, master production scheduling, supply chains, and applied operations research. He has published papers in (and served as a reviewer for) many international journals. 